International Journal of Pure and Applied Mathematics

Volume 84 No. 2 2013, 93-107

ISSN: 1311-8080 (printed version); ISSN: 1314-3395 (on-line version)

url: http://www.ijpam.eu

doi: http://dx.doi.org/10.12732/ijpam.v84i2.8

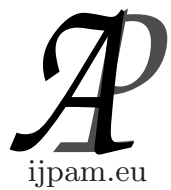

\title{
INTUITIONISTIC FUZZY SOFT ORDERED TERNARY SEMIGROUPS
}

\author{
Muhammad Akram ${ }^{1}$, Naveed Yaqoob ${ }^{2}$ \\ ${ }^{1}$ Department of Mathematics \\ University of Gujrat \\ Gujrat, PAKISTAN \\ ${ }^{2}$ Department of Mathematics \\ Quaid-i-Azam University \\ Islamabad, PAKISTAN
}

\begin{abstract}
In this paper, we introduced the notion of intuitionistic fuzzy soft ideals over an ordered ternary semigroup and their basic properties are investigated.
\end{abstract}

AMS Subject Classification: 20N10, 20M12

Key Words: ordered ternary semigroups, intuitionistic fuzzy soft sets, intuitionistic fuzzy soft ideals

\section{Introduction}

Lehmer [1], studied certain ternary algebraic structures called triplexes in 1932. The notion of ternary semigroup was introduced by Banach. Sioson [2], worked on the ideal theory in ternary semigroups. Many authors worked on ideal theory, fuzzy and intuitionistic fuzzy ideals theory in ordered semigroups and ordered ternary semigroups, see $[3,4,5,6]$.

In 1965, Zadeh [7], introduced the concept of fuzzy sets. In 1986, Atanassov [8], introduced the notion of intuitionistic fuzzy sets which is a generalization of fuzzy sets.

Received: February 1, 2013

(C) 2013 Academic Publications, Ltd. url: www.acadpubl.eu

${ }^{\S}$ Correspondence author 
Molodtsov [9], initiated the concept of soft set theory in 1999 and used this concept for the modeling of uncertainty. Maji et al. [10], defined some binary operations on soft sets, which were later corrected by Ali et al. [11]. Shabir and Ali [12], introduced the notion of soft semigroups. Also see [13].

The notion of fuzzy soft set was introduced by Maji et al. [14]. They studied the union, intersection, compliment and De Morgan Laws for fuzzy soft sets. The results of Maji were further improved by Ahmad and Kharal [15]. Zhou et al. [16], applied the concept of intuitionistic fuzzy soft sets to semigroups and introduced the notion of intuitionistic fuzzy soft ideals over semigroups.

The purpose of this paper is to extend the concept of intuitionistic fuzzy soft set to the theory of ordered ternary semigroup. We introduce the notion of intuitionistic fuzzy soft left (right, lateral) ideals over an ordered ternary semigroup.

\section{Preliminaries}

Definition 1. (see [1]) A ternary semigroup $T$ is a non-empty set whose elements are closed under the ternary operation of multiplication and satisfy the associative law defined as

$$
[[a b c] d e]=[a[b c d] e]=[a b[c d e]], \text { for all } a, b, c, d, e \in T .
$$

For simplicity, we shall write $[a b c]$ as $a b c$. A ternary semigroup $T$ is called an ordered ternary semigroup if there is a partial order $\leq$ on $T$ such that

$$
a \leq b \Rightarrow a c d \leq b c d, c a d \leq c b d, c d a \leq c d b, \text { for all } a, b, c, d \in S .
$$

Let $(T, ., \leq)$ be an ordered ternary semigroup. For $A \subseteq T$, we define

$$
(A]=\{t \in T: t \leq a \text {, for some } a \in A\} .
$$

A non-empty subset $A$ of an ordered ternary semigroup $T$ is called a ternary subsemigroup of $T$ if $(A] \subseteq A$ and $A A A=A^{3} \subseteq A$. By a left (right, lateral) ideal of $T$ we mean a non-empty subset $A$ of $T$ such that $(A] \subseteq A$ and $T T A \subseteq A$ $(A T T \subseteq A, T A T \subseteq A)$ and an ideal is that which is a left, a right and a lateral ideal of $T$. A subsemigroup $A$ of $T$ is called an interior ideal of $T$ if $(A] \subseteq A$ and TTATT $\subseteq A$ and is called a bi-ideal of $T$ if $(A] \subseteq A$ and $A T A T A \subseteq A$.

Definition 2. (see [8]) An intuitionistic fuzzy set (briefly, IFS), $A$ in a non-empty set $X$ is an object having the form,

$$
A=\left\{\left(x, \mu_{A}(x), \gamma_{A}(x)\right) \mid x \in X\right\}
$$


where the functions $\mu_{A}: X \rightarrow[0,1]$ and $\gamma_{A}: X \rightarrow[0,1]$ denotes the degree of membership and the degree of non-membership respectively and for all $x \in X$, $0 \leq \mu_{A}(x)+\gamma_{A}(x) \leq 1$.

An intuitionistic fuzzy set $A=\left\{\left(x, \mu_{A}(x), \gamma_{A}(x)\right) \mid x \in X\right\}$ in $X$ can be identified by an ordered pair $\left(\mu_{A}, \gamma_{A}\right)$ in $I^{X} \times I^{X}$. For simplicity, we shall use IFS for intuitionistic fuzzy set and $A=\left(\mu_{A}, \gamma_{A}\right)$ for IFS

$$
A=\left\{\left(x, \mu_{A}(x), \gamma_{A}(x)\right) \mid x \in X\right\} .
$$

For intuitionistic fuzzy set $A$ and $B, A \subseteq B$, means that $\mu_{A}(x) \leq \mu_{B}(x)$ and $\gamma_{A}(x) \geq \gamma_{B}(x)$, for all $x \in X$. Also the union and intersection is defined as

$$
\begin{aligned}
& A \cup B=\left\{\left(x, \max \left\{\mu_{A}(x), \mu_{B}(x)\right\}, \min \left\{\gamma_{A}(x), \gamma_{B}(x)\right\} \mid x \in X\right)\right\} \\
& A \cap B=\left\{\left(x, \min \left\{\mu_{A}(x), \mu_{B}(x)\right\}, \max \left\{\gamma_{A}(x), \gamma_{B}(x)\right\} \mid x \in X\right)\right\} .
\end{aligned}
$$

For any $r, t \in[0,1], A^{(r, t)}=\left\{x \in X \mid \mu_{A}(x) \geq r\right.$ and $\left.\gamma_{A}(x) \leq t\right\}$ is called the $(r, t)$ - level cut of $A$.

Definition 3. (see [9]) Let $U$ be an initial universe set and $E$ be the set of parameters. Let $P(U)$ denotes the power set of $U$. Let $A$ be a non-empty subset of $E$ then the pair $(F, A)$ is called a soft set over $U$, where $F$ is a mapping given by, $F: A \rightarrow P(U)$.

Definition 4. (see [14]) Let $U$ be an initial universe set and $E$ be the set of parameters. Let $A$ be a non-empty subset of $E$ and $P(F S(U))$ be the collection of all fuzzy subsets of $U$ then the pair $(\widehat{F}, A)$ is called a fuzzy soft set over $U$, where $\widehat{F}$ is a mapping given by, $\widehat{F}: E \rightarrow P(F S(U))$.

Definition 5. (see [17]) Let $U$ be an initial universe set and $E$ be the set of parameters. Let $A$ be a non-empty subset of $E$ and $P(I F S(U))$ be the collection of all intuitionistic fuzzy subsets of $U$ then the pair $(\widetilde{F}, A)$ is called an intuitionistic fuzzy soft set over $U$, where $\widetilde{F}$ is a mapping given by, $\widetilde{F}: A \rightarrow P(\operatorname{IFS}(U))$. In short, we will write $\operatorname{IFSS}(U)$ for intuitionistic fuzzy soft set over $U$.

Definition 6. (see [17]) Let $(\widetilde{F}, A)$ and $(\widetilde{G}, B)$ be two intuitionistic fuzzy soft sets over a common universe $U$ then " $(\widetilde{F}, A)$ AND $(\widetilde{G}, B)$ ", denoted by $(\widetilde{F}, A) \widetilde{\wedge}(\widetilde{G}, B)$ is defined as $(\widetilde{F}, A) \widetilde{\wedge}(\widetilde{G}, B)=(\widetilde{H}, C)$, where $C=A \times B$ and $\widetilde{H}(a, b)=\widetilde{F}(a) \cap \widetilde{G}(b)$, for all $(a, b) \in C=A \times B$.

Definition 7. (see [17]) Let $(\widetilde{F}, A)$ and $(\widetilde{G}, B)$ be two intuitionistic fuzzy soft sets over a common universe $U$ then " $(\widetilde{F}, A)$ OR $(\widetilde{G}, B)$ ", denoted by $(\widetilde{F}, A) \widetilde{V}(\widetilde{G}, B)$ is defined as $(\widetilde{F}, A) \widetilde{V}(\widetilde{G}, B)=(\widetilde{K}, C)$, where $C=A \times B$ and $\widetilde{K}(a, b)=\widetilde{F}(a) \cup \widetilde{G}(b)$, for all $(a, b) \in C=A \times B$. 
Definition 8. (see $[17])$ Let $(\widetilde{F}, A)$ and $(\widetilde{G}, B)$ be two intuitionistic fuzzy soft sets over a common universe $U$ then their union is an intuitionistic fuzzy soft set over $U$ denoted by $(\widetilde{F}, A) \widetilde{\cup}(\widetilde{G}, B)$ and is defined as $(\widetilde{F}, A) \widetilde{U}$ $(\widetilde{G}, B)=(\widetilde{H}, C)$, where $C=A \cup B$ and

$$
\widetilde{H}(c)=\left\{\begin{array}{ll}
\widetilde{F}(c) & \text { if } c \in A-B \\
\widetilde{G}(c) & \text { if } c \in B-A \\
\max \{\widetilde{F}(c), \widetilde{G}(c)\} & \text { if } c \in A \cap B
\end{array} \quad \text { for all } c \in C\right.
$$

Definition 9. (see [17]) Let $(\widetilde{F}, A)$ and $(\widetilde{G}, B)$ be two intuitionistic fuzzy soft sets over a common universe $U$ then their inter section is an intuitionistic fuzzy soft sets over $U$ denoted by $(\widetilde{F}, A) \widetilde{\cap}(\widetilde{G}, B)$ and is defined as $(\widetilde{F}, A) \widetilde{\cap}$ $(\widetilde{G}, B)=(\widetilde{H}, C)$, where $C=A \cup B$ and

$$
\widetilde{H}(c)=\left\{\begin{array}{ll}
\widetilde{F}(c) & \text { if } c \in A-B \\
\widetilde{G}(c) & \text { if } c \in B-A \\
\min \{\widetilde{F}(c), \widetilde{G}(c)\} & \text { if } c \in A \cap B
\end{array} \quad \text { for all } c \in C\right.
$$

Except above definitions of union and intersection of intuitionistic fuzzy soft sets, we may some times use another definitions of union and intersection given as follows.

Definition 10. (see $[16])$ Let $(\widetilde{F}, A)$ and $(\widetilde{G}, B)$ be two intuitionistic fuzzy soft sets over a common universe $U$ such that $A \cap B \neq \phi$. The bi-union of $(\widetilde{F}, A)$ and $(\widetilde{G}, B)$ is defined to be an intuitionistic fuzzy soft set $(\widetilde{H}, C)$ over $U$, where $C=A \cap B$ and $\widetilde{H}(c)=\widetilde{F}(c) \cup \widetilde{G}(c)$ for all $c \in C$. This is denoted by $(\widetilde{H}, C)=(\widetilde{F}, A) \widetilde{\sqcup}(\widetilde{G}, B)$.

Definition 11. (see [16]) Let $(\widetilde{F}, A)$ and $(\widetilde{G}, B)$ be two intuitionistic fuzzy soft sets over a common universe $U$ such that $A \cap B \neq \phi$. The bi-intersection of $(\widetilde{F}, A)$ and $(\widetilde{G}, B)$ is defined to be an intuitionistic fuzzy soft set $(\widetilde{H}, C)$ over $U$, where $C=A \cap B$ and $\widetilde{H}(c)=\widetilde{F}(c) \cap \widetilde{G}(c)$ for all $c \in C$. This is denoted by $(\widetilde{H}, C)=(\widetilde{F}, A) \widetilde{\Pi}(\widetilde{G}, B)$.

If $\left\{\left(\widetilde{F}_{i}, A_{i}\right): i \in I\right\}$ be a collection of intuitionistic fuzzy soft sets over a common universe $U$ such that $\bigcap_{i \in I} A_{i} \neq \phi$, then, we can write $\underset{i \in I}{\widetilde{U}}\left(\widetilde{F}_{i}, A_{i}\right)$ and $\widetilde{\prod}_{i \in I}\left(\widetilde{F}_{i}, A_{i}\right)$. 


\section{Intuitionistic Fuzzy Soft Ideals over Ordered Ternary Semigroup}

In what follows, let $T$ denotes an ordered ternary semigroup unless otherwise specified.

Definition 12. An intuitionistic fuzzy soft set $(\widetilde{F}, A)$ over an ordered ternary semigroup $T$ is called an intuitionistic fuzzy soft ternary subsemigroup over $T$ if

$$
\begin{aligned}
& x \leq y \text { implies } \mu_{\widetilde{F}(a)}(x) \geq \mu_{\widetilde{F}(a)}(y), \gamma_{\widetilde{F}(a)}(x) \leq \gamma_{\widetilde{F}(a)}(y) \\
& \text { and } \mu_{\widetilde{F}(a)}(x z y) \geq \min \left\{\mu_{\widetilde{F}(a)}(x), \mu_{\widetilde{F}(a)}(z), \mu_{\widetilde{F}(a)}(y)\right\}, \\
& \gamma_{\widetilde{F}(a)}(x z y) \leq \max \left\{\gamma_{\widetilde{F}(a)}(x), \gamma_{\widetilde{F}(a)}(z), \gamma_{\widetilde{F}(a)}(y)\right\},
\end{aligned}
$$

for all $x, y, z \in T$ and $a \in A$.

Definition 13. An intuitionistic fuzzy soft set $(\widetilde{F}, A)$ over an ordered ternary semigroup $T$ is called an intuitionistic fuzzy soft left (right, lateral) ideal over $T$ if

$$
\begin{aligned}
x \leq y \text { implies } \mu_{\widetilde{F}(a)}(x) \geq \mu_{\widetilde{F}(a)}(y), \gamma_{\widetilde{F}(a)}(x) \leq \gamma_{\widetilde{F}(a)}(y) \\
\text { and } \mu_{\widetilde{F}(a)}(x z y) \geq \mu_{\widetilde{F}(a)}(y)\left(\begin{array}{c}
\mu_{\widetilde{F}(a)}(x z y) \geq \mu_{\widetilde{F}(a)}(x), \\
\mu_{\widetilde{F}(a)}(x z y) \geq \mu_{\widetilde{F}(a)}(z)
\end{array}\right) \\
\gamma_{\widetilde{F}(a)}(x z y) \leq \gamma_{\widetilde{F}(a)}(y)\left(\begin{array}{c}
\gamma_{\widetilde{F}(a)}(x z y) \leq \gamma_{\widetilde{F}(a)}(x), \\
\gamma_{\widetilde{F}(a)}(x z y) \leq \gamma_{\widetilde{F}(a)}(z)
\end{array}\right),
\end{aligned}
$$

for all $x, y, z \in T$ and $a \in A$.

Definition 14. An intuitionistic fuzzy soft set $(\widetilde{F}, A)$ over an ordered ternary semigroup $T$ is called an intuitionistic fuzzy soft ideal over $T$ if it is an intuitionistic fuzzy soft left, soft right and soft lateral ideal over $T$.

Alternatively we can define,

Definition 15. An intuitionistic fuzzy soft set $(\widetilde{F}, A)$ over an ordered ternary semigroup $T$ is called an intuitionistic fuzzy soft ideal over $T$ if

$$
\begin{aligned}
& x \leq y \text { implies } \mu_{\widetilde{F}(a)}(x) \geq \mu_{\widetilde{F}(a)}(y), \gamma_{\widetilde{F}(a)}(x) \leq \gamma_{\widetilde{F}(a)}(y) \\
& \text { and } \mu_{\widetilde{F}(a)}(x z y) \geq \max \left\{\mu_{\widetilde{F}(a)}(x), \mu_{\widetilde{F}(a)}(z), \mu_{\widetilde{F}(a)}(y)\right\}
\end{aligned}
$$




$$
\gamma_{\widetilde{F}(a)}(x z y) \leq \min \left\{\gamma_{\widetilde{F}(a)}(x), \gamma_{\widetilde{F}(a)}(z), \gamma_{\widetilde{F}(a)}(y)\right\}
$$

for all $x, y, z \in T$ and $a \in A$.

Definition 16. An intuitionistic fuzzy soft ternary subsemigroup $(\widetilde{F}, A)$ of $T$ is called an intuitionistic fuzzy soft interior ideal over $T$ if

$$
\begin{aligned}
& x \leq y \text { implies } \mu_{\widetilde{F}(a)}(x) \geq \mu_{\widetilde{F}(a)}(y), \gamma_{\widetilde{F}(a)}(x) \leq \gamma_{\widetilde{F}(a)}(y) \\
& \text { and } \mu_{\widetilde{F}(a)}(x y z l m) \geq \mu_{\widetilde{F}(a)}(z), \gamma_{\widetilde{F}(a)}(x y z l m) \leq \gamma_{\widetilde{F}(a)}(z),
\end{aligned}
$$

for all $x, y, z, l, m \in T$ and $a \in A$.

Definition 17. An intuitionistic fuzzy soft ternary subsemigroup $(\widetilde{F}, A)$ of $T$ is called an intuitionistic fuzzy soft bi-ideal over $T$ if

$$
\begin{aligned}
x \leq y \text { implies } \mu_{\widetilde{F}(a)}(x) \geq \mu_{\widetilde{F}(a)}(y), \gamma_{\widetilde{F}(a)}(x) \leq \gamma_{\widetilde{F}(a)}(y) & \\
\text { and } \mu_{\widetilde{F}(a)}(x l z m y) & \geq \min \left\{\mu_{\widetilde{F}(a)}(x), \mu_{\widetilde{F}(a)}(z), \mu_{\widetilde{F}(a)}(y)\right\}, \\
\gamma_{\widetilde{F}(a)}(x l z m y) & \leq \max \left\{\gamma_{\widetilde{F}(a)}(x), \gamma_{\widetilde{F}(a)}(z), \gamma_{\widetilde{F}(a)}(y)\right\},
\end{aligned}
$$

for all $x, y, z, l, m \in T$ and $a \in A$.

Lemma 1. An intuitionistic fuzzy soft set $(\widetilde{F}, A)$ over an ordered ternary semigroup $T$ is an intuitionistic fuzzy soft ideal over $T$ if and only if $\widetilde{F}(a)^{(r, t)}$ is an ideal of $T$ for all $r \in(0,1], t \in[1,0)$ and $a \in A$.

Proof. The proof is straightforward.

Theorem 1. Let $(\widetilde{F}, A)$ and $(\widetilde{G}, B)$ be two intuitionistic fuzzy soft ternary subsemigroups (resp. left ideals, right ideals, lateral ideals, ideals) over an ordered ternary semigroup $T$ then $(\widetilde{F}, A) \widetilde{\wedge}(\widetilde{G}, B)$ and $(\widetilde{F}, A) \widetilde{\sqcap}(\widetilde{G}, B)$ are also intuitionistic fuzzy soft ternary subsemigroups (resp. left ideals, right ideals, lateral ideals, ideals) over $T$.

Proof. Let $(\widetilde{F}, A)$ and $(\widetilde{G}, B)$ be two intuitionistic fuzzy soft left ideals over an ordered ternary semigroup $T$, then as defined $(\widetilde{F}, A) \widetilde{\wedge}(\widetilde{G}, B)=(\widetilde{H}, C)$, where $C=A \times B$ and $\widetilde{H}(a, b)=\widetilde{F}(a) \cap \widetilde{G}(b)$, for all $(a, b) \in C=A \times B$. As $(\widetilde{F}, A)$ and $(\widetilde{G}, B)$ are intuitionistic fuzzy soft left ideals over $T$ then for $(a, b) \in C=A \times B$ and $x, y \in T$ such that $x \leq y$, then

$$
\mu_{\widetilde{F}(a)}(x) \geq \mu_{\widetilde{F}(a)}(y), \gamma_{\widetilde{F}(a)}(x) \leq \gamma_{\widetilde{F}(a)}(y)
$$




$$
\text { and } \mu_{\widetilde{G}(b)}(x) \geq \mu_{\widetilde{G}(b)}(y), \gamma_{\widetilde{G}(b)}(x) \leq \gamma_{\widetilde{G}(b)}(y),
$$

implies that

$$
\mu_{\widetilde{F}(a) \cap \widetilde{G}(b)}(x) \geq \mu_{\widetilde{F}(a) \cap \widetilde{G}(b)}(y) \text { and } \gamma_{\widetilde{F}(a) \cap \widetilde{G}(b)}(x) \leq \gamma_{\widetilde{F}(a) \cap \widetilde{G}(b)}(y) .
$$

Thus $\mu_{\widetilde{H}(a, b)}(x) \geq \mu_{\widetilde{H}(a, b)}(y)$ and $\gamma_{\widetilde{H}(a, b)}(x) \leq \gamma_{\widetilde{H}(a, b)}(y)$.

Also for all $x, y, z \in T$ and $(a, b) \in C=A \times B$.

$$
\begin{aligned}
\mu_{\widetilde{H}(a, b)}(x z y) & =\left(\mu_{\widetilde{F}(a)} \cap \mu_{\widetilde{G}(b)}\right)(x z y)=\min \left\{\mu_{\widetilde{F}(a)}(x z y), \mu_{\widetilde{G}(b)}(x z y)\right\} \\
& \geq \min \left\{\mu_{\widetilde{F}(a)}(y), \mu_{\widetilde{G}(b)}(y)\right\}=\left(\mu_{\widetilde{F}(a)} \cap \mu_{\widetilde{G}(b)}\right)(y) \\
& =\mu_{\widetilde{H}(a, b)}(y) .
\end{aligned}
$$

And

$$
\begin{aligned}
\gamma_{\widetilde{H}(a, b)}(x z y) & =\left(\gamma_{\widetilde{F}(a)} \cup \gamma_{\widetilde{G}(b)}\right)(x z y)=\max \left\{\gamma_{\widetilde{F}(a)}(x z y), \gamma_{\widetilde{G}(b)}(x z y)\right\} \\
& \leq \max \left\{\mu_{\widetilde{F}(a)}(y), \mu_{\widetilde{G}(b)}(y)\right\}=\left(\mu_{\widetilde{F}(a)} \cup \mu_{\widetilde{G}(b)}\right)(y) \\
& =\gamma_{\widetilde{H}(a, b)}(y) .
\end{aligned}
$$

Hence, $(\widetilde{F}, A) \widetilde{\wedge}(\widetilde{G}, B)$ is an intuitionistic fuzzy soft left ideal over $T$. The other cases can be proved in a similar way.

Theorem 2. Let $(\widetilde{F}, A)$ and $(\widetilde{G}, B)$ be two intuitionistic fuzzy soft biideals (interior ideals) over an ordered ternary semigroup $T$ then $(\widetilde{F}, A) \widetilde{\wedge}(\widetilde{G}, B)$ and $(\widetilde{F}, A) \widetilde{\sqcap}(\widetilde{G}, B)$ are also intuitionistic fuzzy soft bi-ideals (interior ideals) over $T$.

Proof. Let $(\widetilde{F}, A)$ and $(\widetilde{G}, B)$ be two intuitionistic fuzzy soft bi-ideals over $T$, then they are also intuitionistic fuzzy soft ternary subsemigroups, so by Theorem $1,(\widetilde{F}, A) \widetilde{\wedge}(\widetilde{G}, B)$ is also an intuitionistic fuzzy soft ternary subsemigroup. Since, $(\widetilde{F}, A) \widetilde{\wedge}(\widetilde{G}, B)=(\widetilde{H}, C)$, where $C=A \times B$ and $\widetilde{H}(a, b)=\widetilde{F}(a) \cap \widetilde{G}(b)$. Let $x, y \in T$ such that $x \leq y$ then

$$
\begin{aligned}
\mu_{\widetilde{F}(a)}(x) & \geq \mu_{\widetilde{F}(a)}(y), \gamma_{\widetilde{F}(a)}(x) \leq \gamma_{\widetilde{F}(a)}(y) \\
\text { and } \mu_{\widetilde{G}(b)}(x) & \geq \mu_{\widetilde{G}(b)}(y), \gamma_{\widetilde{G}(b)}(x) \leq \gamma_{\widetilde{G}(b)}(y),
\end{aligned}
$$

implies that

$$
\mu_{\widetilde{F}(a) \cap \widetilde{G}(b)}(x) \geq \mu_{\widetilde{F}(a) \cap \widetilde{G}(b)}(y) \text { and } \gamma_{\widetilde{F}(a) \cap \widetilde{G}(b)}(x) \leq \gamma_{\widetilde{F}(a) \cap \widetilde{G}(b)}(y) .
$$


Thus $\mu_{\widetilde{H}(a, b)}(x) \geq \mu_{\widetilde{H}(a, b)}(y)$ and $\gamma_{\widetilde{H}(a, b)}(x) \leq \gamma_{\widetilde{H}(a, b)}(y)$.

Also for $x, y, z, l, m \in T$ and $(a, b) \in C=A \times B$, we have

$$
\begin{aligned}
& \mu_{\widetilde{H}(a, b)}(x l z m y)=\left(\mu_{\widetilde{F}(a)} \cap \mu_{\widetilde{G}(b)}\right)(x l z m y) \\
& =\min \left\{\mu_{\widetilde{F}(a)}(x l z m y), \mu_{\widetilde{G}(b)}(x l z m y)\right\} \\
& \geq \min \left\{\begin{array}{c}
\max \left\{\mu_{\widetilde{F}(a)}(x), \mu_{\widetilde{F}(a)}(z), \mu_{\widetilde{F}(a)}(y)\right\}, \\
\max \left\{\mu_{\widetilde{G}(b)}(x), \mu_{\widetilde{G}(b)}(z), \mu_{\widetilde{G}(b)}(y)\right\}
\end{array}\right\} \\
& =\max \left\{\begin{array}{c}
\min \left\{\mu_{\widetilde{F}(a)}(x), \mu_{\widetilde{G}(b)}(x)\right\}, \min \left\{\mu_{\widetilde{F}(a)}(z), \mu_{\widetilde{G}(b)}(z)\right\}, \\
\min \left\{\mu_{\widetilde{F}(a)}(y), \mu_{\widetilde{G}(b)}(y)\right\}
\end{array}\right\} \\
& \left.\left.\left.=\max \left\{\left(\mu_{\widetilde{F}(a)} \cap \mu_{\widetilde{G}(b)}\right)(x)\right),\left(\mu_{\widetilde{F}(a)} \cap \mu_{\widetilde{G}(b)}\right)(z)\right\},\left(\mu_{\widetilde{F}(a)} \cap \mu_{\widetilde{G}(b)}\right)(y)\right)\right\} \\
& =\max \left\{\mu_{\widetilde{H}(a, b)}(x), \mu_{\widetilde{H}(a, b)}(z), \mu_{\widetilde{H}(a, b)}(y)\right\},
\end{aligned}
$$

and

$$
\begin{aligned}
& \gamma_{\widetilde{H}(a, b)}(x l z m y)=\left(\gamma_{\widetilde{F}(a)} \cup \gamma_{\widetilde{G}(b)}\right)(x l z m y) \\
& =\max \left\{\gamma_{\widetilde{F}(a)}(x l z m y), \gamma_{\widetilde{G}(b)}(x l z m y)\right\} \\
& \leq \max \left\{\begin{array}{c}
\min \left\{\gamma_{\widetilde{F}(a)}(x), \gamma_{\widetilde{F}(a)}(z), \gamma_{\widetilde{F}(a)}(y)\right\}, \\
\min \left\{\gamma_{\widetilde{G}(b)}(x), \gamma_{\widetilde{G}(b)}(z), \gamma_{\widetilde{G}(b)}(y)\right\}
\end{array}\right\} \\
& =\min \left\{\begin{array}{c}
\left.\max \left\{\gamma_{\widetilde{F}(a)}(x), \gamma_{\widetilde{G}(b)}(x)\right\}, \max \left\{\gamma_{\widetilde{F}(a)}(z), \gamma_{\widetilde{G}(b)}(z)\right\}, \quad \max _{\widetilde{F}(a)}(y), \gamma_{\widetilde{G}(b)}(y)\right\} \\
\left.\left.\left.=\min \left\{\left(\gamma_{\widetilde{F}(a)} \cup \gamma_{\widetilde{G}(b)}\right)(x)\right),\left(\gamma_{\widetilde{F}(a)} \cup \gamma_{\widetilde{G}(b)}\right)(z)\right),\left(\gamma_{\widetilde{F}(a)} \cup \gamma_{\widetilde{G}(b)}\right)(y)\right)\right\} \\
=\min \left\{\gamma_{\widetilde{H}(a, b)}(x), \gamma_{\widetilde{H}(a, b)}(z), \gamma_{\widetilde{H}(a, b)}(y)\right\} .
\end{array}\right.
\end{aligned}
$$

Hence $(\widetilde{F}, A) \widetilde{\wedge}(\widetilde{G}, B)$ is an intuitionistic fuzzy soft bi-ideal over $T$. The other cases can be proved in a similar way.

Theorem 3. Let $(\widetilde{F}, A)$ and $(\widetilde{G}, B)$ be two intuitionistic fuzzy soft ternary subsemigroups (resp. left ideals, right ideals, lateral ideals, ideals, bi-ideals, interior ideals) over an ordered ternary semigroup $T$ then $(\widetilde{F}, A) \widetilde{V}(\widetilde{G}, B)$ and $(\widetilde{F}, A) \widetilde{\sqcup}(\widetilde{G}, B)$ are also intuitionistic fuzzy soft ternary subsemigroups (resp. left ideals, right ideals, lateral ideals, ideals, bi-ideals, interior ideals) over $T$.

Proof. The proof is straightforward.

Theorem 4. Let $(\widetilde{F}, A)$ and $(\widetilde{G}, B)$ be two intuitionistic fuzzy soft ternary subsemigroups (resp. left ideals, right ideals, lateral ideals, ideals) over an ordered ternary semigroup $T$ then $(\widetilde{F}, A) \widetilde{\cap}(\widetilde{G}, B)$ and $(\widetilde{F}, A) \widetilde{\cup}(\widetilde{G}, B)$ are also intuitionistic fuzzy soft ternary subsemigroups (resp. left ideals, right ideals, lateral ideals, ideals) over $T$. 
Proof. Let $(\widetilde{F}, A)$ and $(\widetilde{G}, B)$ be two intuitionistic fuzzy soft left ideals over an ordered ternary semigroup $T$ then $(\widetilde{F}, A) \widetilde{\cap}(\widetilde{G}, B)=(\widetilde{H}, C)$, where $C=A \cup B$ and

$$
\widetilde{H}(c)=\left\{\begin{array}{lr}
\widetilde{F}(c) & \text { if } c \in A-B \\
\widetilde{G}(c) & \text { if } c \in B-A \\
\min \{\widetilde{F}(c), \widetilde{G}(c)\} & \text { if } c \in A \cap B
\end{array}, \text { for all } c \in C .\right.
$$

Let $c \in C$ and $x, y, z \in T$, then we have,

(i) if $c \in A-B$, implies $\widetilde{H}(c)=\widetilde{F}(c)$. Let $x, y \in T$ such that $x \leq y$ then

$$
\begin{aligned}
\mu_{\widetilde{H}(c)}(x) & =\left(\mu_{\widetilde{F}(c)}\right)(x) \geq\left(\mu_{\widetilde{F}(c)}\right)(y)=\mu_{\widetilde{H}(c)}(y) \\
\text { and } \gamma_{\widetilde{H}(c)}(x) & =\left(\gamma_{\widetilde{F}(c)}\right)(x) \leq\left(\gamma_{\widetilde{F}(c)}\right)(y)=\gamma_{\widetilde{H}(c)}(x) .
\end{aligned}
$$

Also for $x, y, z \in T$, we have $\mu_{\widetilde{H}(c)}(x z y)=\mu_{\widetilde{F}(c)}(x z y) \geq \mu_{\widetilde{F}(c)}(y)=\mu_{\widetilde{H}(c)}(y)$. And $\gamma_{\widetilde{H}(c)}(x z y)=\gamma_{\widetilde{F}(c)}(x z y) \leq \gamma_{\widetilde{F}(c)}(x)=\gamma_{\widetilde{H}(c)}(y)$.

(ii) if $c \in B-A$, implies $\widetilde{H}(c)=\widetilde{G}(c)$. Let $x, y \in T$ such that $x \leq y$ then

$$
\begin{aligned}
\mu_{\widetilde{H}(c)}(x) & =\left(\mu_{\widetilde{G}(c)}\right)(x) \geq\left(\mu_{\widetilde{G}(c)}\right)(y)=\mu_{\widetilde{H}(c)}(y) \\
\text { and } \gamma_{\widetilde{H}(c)}(x) & =\left(\gamma_{\widetilde{G}(c)}\right)(x) \leq\left(\gamma_{\widetilde{G}(c)}\right)(y)=\gamma_{\widetilde{H}(c)}(y) .
\end{aligned}
$$

Also for $x, y, z \in T$, we have $\mu_{\widetilde{H}(c)}(x z y)=\mu_{\widetilde{G}(c)}(x z y) \geq \mu_{\widetilde{G}(c)}(y)=\mu_{\widetilde{H}(c)}(y)$. And $\gamma_{\widetilde{H}(c)}(x z y)=\gamma_{\widetilde{G}(c)}(x z y) \leq \gamma_{\widetilde{G}(c)}(y)=\gamma_{\widetilde{H}(c)}(y)$.

(iii) if $c \in A \cap B$, then $\widetilde{H}(c)=\min \{\widetilde{F}(c), \widetilde{G}(c)\}=\widetilde{F}(c) \cap \widetilde{G}(c$.

We can easily verify that for $x, y \in T$ such that $x \leq y$ implies that

$$
\mu_{\widetilde{H}(c)}(x) \geq \mu_{\widetilde{H}(c)}(y) \text { and } \gamma_{\widetilde{H}(c)}(x) \leq \gamma_{\widetilde{H}(c)}(y) .
$$

Also for $x, y, z \in T$, we have $\mu_{\widetilde{H}(c)}(x z y) \geq \mu_{\widetilde{H}(c)}(y)$ and $\gamma_{\widetilde{H}(c)}(x z y) \leq \gamma_{\widetilde{H}(c)}(y)$.

Hence this shows that $(\widetilde{F}, A) \widetilde{\cap}(\widetilde{G}, B)$ is an ntuitionistic fuzzy soft left ideal over $T$. The other cases can be proved in a similar way.

Theorem 5. Let $(\widetilde{F}, A)$ and $(\widetilde{G}, B)$ be two intuitionistic fuzzy soft biideals (interior ideals) over an ordered ternary semigroup $T$, then $(\widetilde{F}, A) \widetilde{\cap}$ $(\widetilde{G}, B)$ and $(\widetilde{F}, A) \widetilde{\cup}(\widetilde{G}, B)$ are also intuitionistic fuzzy soft bi-ideals (interior ideals) over $T$.

Proof. The proof is straightforward. 
Theorem 6. Let $\Delta(T, E)$ be the collection of all intuitionistic fuzzy soft left (resp. right, lateral, bi, interior) ideals over an ordered ternary semigroup $T$. Then $(\Delta(T, E), \widetilde{\cup}, \widetilde{\Pi})$ is a complete distributive lattice under the relation $\widetilde{\simeq}$.

Proof. Let $(\widetilde{F}, A)$ and $(\widetilde{G}, B)$ be two intuitionistic fuzzy soft left ideals over an ordered ternary semigroup $T$, that is $(\widetilde{F}, A),(\widetilde{G}, B) \in \Delta(T, E)$, then by Theorem 1 and Theorem $4,(\widetilde{F}, A) \widetilde{\Pi}(\widetilde{G}, B)$ and $(\widetilde{F}, A) \widetilde{\cup}(\widetilde{G}, B)$ and are intuitionistic fuzzy soft left ideals over $T$, implies that $(\widetilde{F}, A) \widetilde{\cup}(\widetilde{G}, B),(\widetilde{F}, A) \widetilde{\Pi}(\widetilde{G}, B) \in$ $\Delta(T, E)$. Obviously, we can say that $(\widetilde{F}, A) \widetilde{\cup}(\widetilde{G}, B)$ is the least upper bound and $(\widetilde{F}, A) \widetilde{\sqcap}(\widetilde{G}, B)$ is the greatest lower bound of the collection $\{(\widetilde{F}, A),(\widetilde{G}, B)\}$. Hence for any arbitrary collection of $\Delta(T, E)$, there exist a least upper bound and a greatest lower bound, which implies that $\Delta(T, E)$ is a complete lattice. Also for $(\widetilde{F}, A),(\widetilde{G}, B)$ and $(\widetilde{H}, C) \in \Delta(T, E)$, we have

$$
\begin{aligned}
(\widetilde{F}, A) \widetilde{\sqcap}(\widetilde{G}, B) \widetilde{\cup}(\widetilde{H}, C) & =(\widetilde{I}, A \cap(B \cup C)) \text { and } \\
((\widetilde{F}, A) \widetilde{\sqcap}(\widetilde{G}, B)) \widetilde{\cup}((\widetilde{F}, A) \widetilde{\sqcap}(\widetilde{H}, C)) & =(\widetilde{J},(A \cap B) \cup(A \cap C)) \\
& =(\widetilde{J}, A \cap(B \cup C)) .
\end{aligned}
$$

Easily, we can show that for any $z \in A \cap(B \cup C), \widetilde{I}(z)=\widetilde{J}(z)$, which implies that

$$
(\widetilde{F}, A) \widetilde{\sqcap}(\widetilde{G}, B) \widetilde{\cup}(\widetilde{H}, C)=((\widetilde{F}, A) \widetilde{\sqcap}(\widetilde{G}, B)) \widetilde{\cup}((\widetilde{F}, A) \widetilde{\sqcap}(\widetilde{H}, C)) .
$$

Hence $\Delta(T, E)$ is a complete distributive lattice. The other cases can be proved in a similar way.

Theorem 7. Let $\Delta(T, E)$ be the collection of all intuitionistic fuzzy soft biideals (interior ideals) over an ordered ternary semigroup $T$. Then $(\Delta(T, E), \widetilde{\sqcup}, \widetilde{\cap})$ is a complete distributive lattice under the relation $\widetilde{\subseteq}$.

Proof. The proof is straightforward.

Now, consider a specific family of parameters say, $D \subseteq E$. We denote the set of intuitionistic fuzzy soft ideals over an ordered ternary semigroup $T$ with parameter set $D$ as $\Delta_{D}(T)$, where $\Delta_{D}(T)=\{(\widetilde{F}, A) \in \Delta(T, E) \mid \widetilde{F}: D \rightarrow$ $P(I F S(T)\}$.

Lemma 2. Let $(\widetilde{F}, A)$ and $(\widetilde{G}, B) \in \Delta_{D}(T)$, then $(\widetilde{F}, A) \widetilde{\cup}(\widetilde{G}, B) \in \Delta_{D}(T)$ and $(\widetilde{F}, A) \widetilde{\sqcap}(\widetilde{G}, B) \in \Delta_{D}(T)$.

Proof. The proof is straightforward. 
Lemma 3. Let $(\widetilde{F}, A)$ and $(\widetilde{G}, B) \in \Delta_{D}(T)$, then $(\widetilde{F}, A) \widetilde{\cap}(\widetilde{G}, B) \in \Delta_{D}(T)$ and $(\widetilde{F}, A) \widetilde{\sqcup}(\widetilde{G}, B) \in \Delta_{D}(T)$.

Proof. The proof is straightforward.

Definition 18. Let $(\widetilde{F}, A),(\widetilde{G}, B)$ and $(\widetilde{H}, C)$ be three intuitionistic fuzzy soft sets over an ternary semigroup $T$. Then their product is defined as $(\widetilde{F}, A) \circ$ $(\widetilde{G}, B) \circ(\widetilde{H}, C)=(\widetilde{F} \circ \widetilde{G} \circ \widetilde{H}, Z)$, where $Z=A \cup B \cup C$,

$$
\begin{gathered}
\mu_{(\widetilde{F} \circ \widetilde{G} \circ \widetilde{H})(z)}(t) \\
= \begin{cases}\mu_{\widetilde{F}(z)}(t) & \text { if } z \in A-(B \cup C) \\
\mu_{\widetilde{G}(z)}(t) & \text { if } z \in B-(C \cup A) \\
\mu_{\widetilde{H}(z)}(t) & \text { if } z \in C-(A \cup B) \\
\sup _{t=\alpha \beta \delta} \min \left\{\mu_{\widetilde{F}(z)}(\alpha), \mu_{\widetilde{G}(z)}(\beta), \mu_{\widetilde{H}(z)}(\delta)\right\} & \text { if } z \in A \cap B \cap C\end{cases}
\end{gathered}
$$

and

$$
\begin{gathered}
\gamma_{(\widetilde{F} \circ \widetilde{G} \circ \widetilde{H})(z)}(t) \\
= \begin{cases}\gamma_{\widetilde{F}(z)}(t) & \text { if } z \in A-(B \cup C) \\
\gamma_{\widetilde{G}(z)}(t) & \text { if } z \in B-(C \cup A) \\
\gamma_{\widetilde{H}(c)}(t) & \text { if } z \in C-(A \cup B) \\
\inf _{t=\alpha \beta \delta} \max \left\{\gamma_{\widetilde{F}(z)}(\alpha), \gamma_{\widetilde{G}(z)}(\beta), \gamma_{\widetilde{H}(z)}(\delta)\right\} & \text { if } z \in A \cap B \cap C\end{cases}
\end{gathered}
$$

for all $z \in Z$.

Theorem 8. Let $(\widetilde{F}, A),(\widetilde{G}, B)$ and $(\widetilde{H}, C)$ be three intuitionistic fuzzy soft ideals (resp. left ideals, right ideals, lateral ideals, interior ideals, bi-ideals) over an ordered ternary semigroup $T$, then their product $(\widetilde{F}, A) \circ(\widetilde{G}, B) \circ(\widetilde{H}, C)$ is also an intuitionistic fuzzy soft ideal (resp. left ideal, right ideal, lateral ideal, interior ideal, bi-ideal) over $T$.

Proof. Let $(\widetilde{F}, A),(\widetilde{G}, B)$ and $(\widetilde{H}, C)$ be three intuitionistic fuzzy soft ideals over an ordered ternary semigroup $T$. Let $c \in Z$ and $x, y, z \in T$, then we have

(i) if $c \in A-(B \cup C)$ and $x, y \in T$, such that $x \leq y$, then

$$
\mu_{(\widetilde{F} \circ \widetilde{G} \circ \widetilde{H})(c)}(x)=\mu_{(\widetilde{F})(c)}(x) \leq \mu_{(\widetilde{F})(c)}(y)=\mu_{(\widetilde{F} \circ \widetilde{G} \circ \widetilde{H})(c)}(y),
$$

and

$$
\gamma_{(\widetilde{F} \circ \widetilde{G} \circ \widetilde{H})(c)}(x)=\gamma_{(\widetilde{F})(c)}(x) \geq \gamma_{(\widetilde{F})(c)}(y)=\gamma_{(\widetilde{F} \circ \widetilde{G} \circ \widetilde{H})(c)}(y) .
$$


Also for all $x, y, z \in T$,

$$
\begin{aligned}
& \mu_{(\widetilde{F} \circ \widetilde{G} \circ \widetilde{H})(c)}(x y z) \\
= & \mu_{(\widetilde{F})(c)}(x y z) \geq \max \left\{\mu_{(\widetilde{F})(c)}(x), \mu_{(\widetilde{F})(c)}(y), \mu_{(\widetilde{F})(c)}(z)\right\} \\
= & \left.\max _{(\widetilde{F} \circ \widetilde{G} \circ \widetilde{H})(c)}(x), \mu_{(\widetilde{F} \circ \widetilde{G} \circ \widetilde{H})(c)}(y), \mu_{(\widetilde{F} \circ \widetilde{G} \circ \widetilde{H})(c)}(z)\right\} .
\end{aligned}
$$

Also

$$
\begin{aligned}
& \gamma_{(\widetilde{F} \circ \widetilde{G} \circ \widetilde{H})(c)}(x y z) \\
= & \gamma_{(\widetilde{F})(c)}(x y z) \leq \min \left\{\gamma_{(\widetilde{F})(c)}(x), \gamma_{(\widetilde{F})(c)}(y), \gamma_{(\widetilde{F})(c)}(z)\right\} \\
= & \min \left\{\gamma_{(\widetilde{F} \circ \widetilde{G} \circ \widetilde{H})(c)}(x), \gamma_{(\widetilde{F} \circ \widetilde{G} \circ \widetilde{H})(c)}(y), \gamma_{(\widetilde{F} \circ \widetilde{G} \circ \widetilde{H})(c)}(z)\right\} .
\end{aligned}
$$

(ii) If $c \in B-(C \cup A)$, then the proof is similar to (i).

(iii) If $c \in C-(A \cup B)$, then the proof is similar to (i).

(iv) If $c \in A \cap B \cap C$, and $x, y \in T$ with $x \leq y$ then

$$
\mu_{(\widetilde{F} \circ \widetilde{G} \circ \widetilde{H})(c)}(x) \leq \mu_{(\widetilde{F} \circ \widetilde{G} \circ \widetilde{H})(c)}(y) \text { and } \gamma_{(\widetilde{F} \circ \widetilde{G} \circ \widetilde{H})(c)}(x) \geq \gamma_{(\widetilde{F} \circ \widetilde{G} \circ \widetilde{H})(c)}(y)
$$

Also for all $x, y, z \in T$, we have

$$
\begin{aligned}
\mu_{(\widetilde{F} \circ \widetilde{G} \circ \widetilde{H})(c)}(x) & =\sup _{x=\alpha \beta \delta}\left\{\min \left\{\mu_{\widetilde{F}(z)}(\alpha), \mu_{\widetilde{G}(z)}(\beta), \mu_{\widetilde{H}(z)}(\delta)\right\}\right\} \\
& \leq \sup _{x y z=\alpha \beta \delta y z}\left\{\min \left\{\mu_{\widetilde{F}(z)}(\alpha), \mu_{\widetilde{G}(z)}(\beta), \mu_{\widetilde{H}(z)}(\delta y z)\right\}\right\} \\
& \leq \sup _{x y z=u v w}\left\{\min \left\{\mu_{\widetilde{F}(z)}(u), \mu_{\widetilde{G}(z)}(v), \mu_{\widetilde{H}(z)}(w)\right\}\right\} \\
& =\mu_{(\widetilde{F} \circ \widetilde{G} \circ \widetilde{H})(c)}(x y z)
\end{aligned}
$$

$\Rightarrow \mu_{(\widetilde{F} \circ \widetilde{G} \circ \widetilde{H})(c)}(x) \leq \mu_{(\widetilde{F} \circ \widetilde{G} \circ \widetilde{H})(c)}(x y z)$. Similarly, we can show that,

$\mu_{(\widetilde{F} \circ \widetilde{G} \circ \widetilde{H})(c)}(y) \leq \mu_{(\widetilde{F} \circ \widetilde{G} \circ \widetilde{H})(c)}(x y z)$ and $\mu_{(\widetilde{F} \circ \widetilde{G} \circ \widetilde{H})(c)}(z) \leq \mu_{(\widetilde{F} \circ \widetilde{G} \circ \widetilde{H})(c)}(x y z)$.

Which implies that,

$$
\mu_{(\widetilde{F} \circ \widetilde{G} \circ \widetilde{H})(c)}(x y z) \geq \max \left\{\mu_{(\widetilde{F} \circ \widetilde{G} \circ \widetilde{H})(c)}(x), \mu_{(\widetilde{F} \circ \widetilde{G} \circ \widetilde{H})(c)}(y), \mu_{(\widetilde{F} \circ \widetilde{G} \circ \widetilde{H})(c)}(z)\right\} .
$$

Also, it is easy to verify that,

$$
\gamma_{(\widetilde{F} \circ \widetilde{G} \circ \widetilde{H})(c)}(x y z) \geq \min \left\{\gamma_{(\widetilde{F} \circ \widetilde{G} \circ \widetilde{H})(c)}(x), \gamma_{(\widetilde{F} \circ \widetilde{G} \circ \widetilde{H})(c)}(y), \gamma_{(\widetilde{F} \circ \widetilde{G} \circ \widetilde{H})(c)}(z)\right\}
$$

Hence, $(\widetilde{F}, A) \circ(\widetilde{G}, B) \circ(\widetilde{H}, C)$ is an intuitionistic fuzzy soft ideal over $T$. The other cases can be proved in a similar way. 
Theorem 9. Let $T$ be an ordered ternary semigroup with identity $e$ and $\Omega(S, E)$ be the collection of all intuitionistic fuzzy soft ideals over $T$ with the property that $(\widetilde{F}, A) \in \Omega(S, E)$ if and only if $\mu_{\widetilde{F}(z)}(e)=1$ and $\gamma_{\widetilde{F}(z)}(e)=0$ then $(\Omega(S, E), \circ, \widetilde{\Pi})$ is a complete lattice under $\widetilde{\subseteq}$.

Proof. Let $(\widetilde{F}, A),(\widetilde{G}, B)$ and $(\widetilde{H}, C) \in \Omega(S, E)$ then $\mu_{\widetilde{F}(z)}(e)=\mu_{\widetilde{G}(z)}(e)=$ $\mu_{\widetilde{H}(z)}(e)=1$ and $\gamma_{\widetilde{F}(z)}(e)=\gamma_{\widetilde{G}(z)}(e)=\gamma_{\widetilde{G}(z)}(e)=0$. As $(\widetilde{F}, A),(\widetilde{G}, B)$ and $(\widetilde{H}, C)$ are intuitionistic fuzzy soft ideals over $T$ then so is

$$
(\widetilde{F}, A) \widetilde{\sqcap}(\widetilde{G}, B) \widetilde{\sqcap}(\widetilde{H}, C)
$$

and

$$
(\widetilde{F}, A) \circ(\widetilde{G}, B) \circ(\widetilde{H}, C) .
$$

As $\mu_{(\widetilde{F} \cap \widetilde{G} \cap \widetilde{G})(z)}(e)=1$ and $\gamma_{(\widetilde{F} \cap \widetilde{G} \cap \widetilde{G})(z)}(e)=0$. Also $\mu_{(\widetilde{F} \circ \widetilde{G} \circ \widetilde{G})(z)}(e)=1$ and $\gamma_{(\widetilde{F} \circ \widetilde{G} \circ \widetilde{G})(z)}(e)=0$. Which implies that $(\widetilde{F}, A) \widetilde{\sqcap}(\widetilde{G}, B) \widetilde{\sqcap}(\widetilde{H}, C)$ and $(\widetilde{F}, A) \circ$ $(\widetilde{G}, B) \circ(\widetilde{H}, C) \in \Omega(S, E)$. Note that $(\widetilde{F}, A) \widetilde{\sqcap}(\widetilde{G}, B) \widetilde{\Pi}(\widetilde{H}, C)$ is the greatest lower bound of the class $\{(\widetilde{F}, A),(\widetilde{G}, B),(\widetilde{H}, C)\}$. Now for least upper bound, let $z \in A \cup B \cup C$ and $x \in T$ then, we have

(i) If $z \in A-(B \cup C)$, then by definition, $\mu_{(\widetilde{F} \circ \widetilde{G} \circ \widetilde{G})(z)}(x)=\mu_{(\widetilde{F})(z)}(x)$ and $\gamma_{(\widetilde{F} \circ \widetilde{G} \circ \widetilde{G})(z)}(x)=\gamma_{(\widetilde{F})(z)}(x)$.

(ii) If $z \in B-(A \cup C)$, then $\mu_{(\widetilde{F} \circ \widetilde{G} \circ \widetilde{G})(z)}(x)=\mu_{(\widetilde{G})(z)}(x)$ and $\gamma_{(\widetilde{F} \circ \widetilde{G} \circ \widetilde{G})(z)}(x)=$ $\gamma_{(\widetilde{G})(z)}(x)$.

(iii) If $z \in C-(A \cup B)$, then $\mu_{(\widetilde{F} \circ \widetilde{G} \circ \widetilde{G})(z)}(x)=\mu_{(\widetilde{H})(z)}(x)$ and $\gamma_{(\widetilde{F} \circ \widetilde{G} \circ \widetilde{G})(z)}(x)=$ $\gamma_{(\widetilde{H})(z)}(x)$.

(iv) If $z \in A \cap B \cap C$, then

$$
\begin{aligned}
\mu_{(\widetilde{F} \circ \widetilde{G} \circ \widetilde{H})(c)}(x) & =\sup _{x=x e e} \min \left\{\mu_{\widetilde{F}(z)}(x), \mu_{\widetilde{G}(z)}(e), \mu_{\widetilde{H}(z)}(e)\right\} \\
& \geq \min \left\{\mu_{\widetilde{F}(z)}(x), \mu_{\widetilde{G}(z)}(e), \mu_{\widetilde{H}(z)}(e)\right\} \\
& =\mu_{\widetilde{F}(z)}(x), \text { since } \mu_{\widetilde{G}(z)}(e)=\mu_{\widetilde{H}(z)}(e)=1
\end{aligned}
$$

and

$$
\begin{aligned}
\gamma_{(\widetilde{F} \circ \widetilde{G} \circ \widetilde{H})(c)}(x) & =\inf _{x=x e e} \max \left\{\gamma_{\widetilde{F}(z)}(x), \gamma_{\widetilde{G}(z)}(e), \gamma_{\widetilde{H}(z)}(e)\right\} \\
& \leq \max \left\{\gamma_{\widetilde{F}(z)}(x), \gamma_{\widetilde{G}(z)}(e), \gamma_{\widetilde{H}(z)}(e)\right\} \\
& =\gamma_{\widetilde{F}(z)}(x), \text { since } \gamma_{\widetilde{G}(z)}(e)=\gamma_{\widetilde{H}(z)}(e)=0 .
\end{aligned}
$$


Which implies that $(\widetilde{F}, A) \widetilde{\subseteq}(\widetilde{F}, A) \circ(\widetilde{G}, B) \circ(\widetilde{H}, C)$. Similarly, we can show that $(\widetilde{G}, B) \widetilde{\subseteq}(\widetilde{F}, A) \circ(\widetilde{G}, B) \circ(\widetilde{H}, C)$ and $(\widetilde{H}, C) \widetilde{\subseteq}(\widetilde{F}, A) \circ(\widetilde{G}, B) \circ(\widetilde{H}, C)$, implies that $(\widetilde{F}, A) \circ(\widetilde{G}, B) \circ(\widetilde{H}, C)$ is an upper bound of $\{(\widetilde{F}, A),(\widetilde{G}, B),(\widetilde{H}, C)\}$. Now, let $(\widetilde{K}, D) \in \Omega(S, E)$ such that $(\widetilde{F}, A) \subseteq(\widetilde{K}, D),(\widetilde{G}, B) \subseteq(\widetilde{K}, D)$ and $(\underset{\widetilde{H}}{\widetilde{K}}, C) \subseteq(\widetilde{K}, D)$. Then $(\widetilde{F}, A) \circ(\widetilde{G}, B) \circ(\widetilde{H}, C) \subseteq(\widetilde{K}, D) \circ(\widetilde{K}, D) \circ(\widetilde{K}, D) \subseteq$ $(\widetilde{K}, D)$. Hence $(\widetilde{F}, A) \circ(\widetilde{G}, B) \circ(\widetilde{H}, C)$ is a least upper bound of the class $\{(\widetilde{F}, A),(\widetilde{G}, B),(\widetilde{H}, C)\}$. Since $\{(\widetilde{F}, A),(\widetilde{G}, B),(\widetilde{H}, C)\}$ is an orbitrary subclass of $\Omega(S, E)$. Hence $(\Omega(S, E), \circ, \widetilde{\Pi})$ is a complete lattice.

\section{References}

[1] D.H. Lehmer, A ternary analogue of abelian groups, Amer. J. Math., 54, No. 2 (1932), 329-338.

[2] F.M. Sioson, Ideal theory in ternary semigroups, Math. Japan., 10 (1965) 63-84.

[3] A. Khan, M. Khan, S. Hussain, Intuitionistic fuzzy ideals in ordered semigroups, J. Appl. Math. Inform., 28, No. (1-2) (2010), 311-324.

[4] R. Chinram, S. Saelee, Fuzzy ideals and fuzzy filters of ordered ternary semigroups, J. Math. Res., 2, No. 1 (2010), 93-97.

[5] K.H. Kim, Intuitionistic fuzzy semiprime ideals of ordered semigroups, J. Chungcheong Math. Soc., 22, No. 2 (2009), 235-243.

[6] N. Yaqoob, S. Abdullah, N. Rehman, M. Naeem, Roughness and fuzziness in ordered ternary semigroups, World Appl. Sci. J., 17, No. 12 (2012), 1683-1693.

[7] L.A. Zadeh, Fuzzy sets, Inform. Control, 8 (1965), 338-353.

[8] K.T. Atanassov, Intuitionistic fuzzy sets, Fuzzy Sets and Systems, 20 (1986), 87-96.

[9] D. Molodtsov, Soft set theory-First results, Comput. Math. Appl., 37 (1999), 19-31.

[10] P.K. Maji, A.R. Roy, R. Biswas, Soft set theory, Comput. Math. Appl., 45 (2003), 555-562.

[11] M.I. Ali, F. Feng, X.Y. Liu, M. Shabir, On some new operations in soft set theory, Comput. Math. Appl., 57 (2009), 1547-1553. 
[12] M. Shabir, M.I. Ali, Soft ideals and generalized fuzzy ideals in semigroups, New Math. Nat. Comput., 5 (2009), 599-615.

[13] M. Shabir, A. Ahmad, On soft ternary semigroups, Ann. Fuzzy Math. Inform., 3 (2012), 39-59.

[14] P.K. Maji, A.R. Roy, R.Biswas, Fuzzy soft sets, J. Fuzzy Math., 9, No. 3 (2001), 589-602.

[15] B. Ahmad, A. Kharal, On fuzzy soft sets, Adv. Fuzzy Syst., (2009) 1-6.

[16] J. Zhou, Y. Li, Y. Yin, Intuitionistic fuzzy soft semigroups, Math. Aeterna, 1, No. 3 (2011), 173-183.

[17] P.K. Maji, A.R. Roy, R. Biswas, Intuitionistic fuzzy soft sets, J. Fuzzy Math., 9, No. 3 (2001), 677-692. 
